\title{
Scattering theory of the screened Casimir interaction in electrolytes
}

\author{
P. A. Maia Neto, ${ }^{1}$ F. S. S. Rosa ${ }^{1}$ L. B. Pires,${ }^{1}$ A. B. Marim,${ }^{1}$ A. \\ Canaguier-Durand, ${ }^{2}$ R. Guérout, ${ }^{2}$ A. Lambrecht, ${ }^{2}$ and S. Reynaud ${ }^{2}$ \\ ${ }^{1}$ Instituto de Física, Universidade Federal do Rio de Janeiro, \\ CP 68528, Rio de Janeiro RJ 21941-909, Brazil \\ ${ }^{2}$ Laboratoire Kastler Brossel, Sorbonne Université, CNRS, ENS-PSL Université, \\ Collège de France, Campus Pierre et Marie Curie, F-75252 Paris, France
}

(Dated: June 18, 2019)

\begin{abstract}
We apply the scattering approach to the Casimir interaction between two dielectric half-spaces separated by an electrolyte solution. We take the nonlocal electromagnetic response of the intervening medium into account, which results from the presence of movable ions in solution. In addition to the usual transverse modes, we consider longitudinal channels and their coupling by reflection at the surface of the local dielectric. The Casimir interaction energy is calculated from the matrix describing a round-trip of coupled transverse and longitudinal waves between the interacting surfaces. The nonzero-frequency contributions are approximately unaffected by the presence of ions. We find, at zero frequency, a contribution from longitudinal channels, which is screened over a distance of the order of the Debye length, alongside an unscreened term arising from transverse-magnetic modes. The latter defines the long-distance asymptotic limit for the interaction.
\end{abstract}

\section{INTRODUCTION}

Over the last decade, the scattering approach [1, 2] to the Casimir effect 3 has allowed for the derivation of exact results for a number of non-trivial geometries, including the ones most often investigated experimentally: the plane-sphere [4-11] and sphere-sphere [12 14]. Within the scattering approach, the Casimir effect arises from the recurrent multiple scattering of electromagnetic fluctuations between the interacting surfaces [15]. In the particular case of two homogenous half-spaces separated by a layer of empty space or of a third homogeneous medium, one recovers the standard Lifshitz [16] and Dzyaloshinskii-Lifshitz-Pitaevskii (DLP) 17] results, respectively. The unretarded van der Waals interaction is obtained in the limit of short distances as a particular case $[18$.

Applications of the scattering approach in colloid sciences and biophysics requires the inclusion of the screening caused by ions dissolved in a polar liquid (water in most cases). As in the double-layer interaction between prescribed charged surfaces [19, 20], movable ions could indeed be expected to screen slowly fluctuating charges. Alternatively, in the language of the scattering approach, the electrolyte solution displays a nonlocal electric response (spatial dispersion) allowing for the existence of longitudinal modes [21] in addition to the standard transverse ones.

The complementary case in which the intervening medium is local, whereas the interacting half-spaces are nonlocal, has been extensively analyzed in connection with the anomalous skin depth effect in metals. Indeed, free electrons exhibit a nonlocal response that modifies the Casimir interaction between metallic plates 22 26]. The nonlocal response of metals has been recently considered in connection with quantum friction [27]. The unretarded van der Waals interaction between nonlocal half-spaces across a local medium has been derived in the context of metals [28] and electrolytes [21].

In the case of electrolytes, the common view is that only the Matsubara zero-frequency contribution as given by DLP result [17] is modified by screening (see for instance [29] for a recent review), since the plasma frequency associated to the presence of ions is always much smaller than $k_{B} T / \hbar$ (where $T$ is the temperature). In other words, fluctuations at all nonzero Matsubara frequencies are too fast to be screened by ions in solution. The zero-frequency contribution is then usually considered apart from the nonzero Matsubara terms, and results are derived from the linear Poisson-Boltzmann equation, either by considering its Green function 30, or by analyzing the zero-point energy of surface modes [31, 32 as in Ref. 33.

For the interaction between metallic plates, the zero frequency contribution is relevant only at very long distances, in the micrometer range and beyond. Screening is hence negligible in the experiments probing the Casimir force between metallic surfaces across ethanol 34, 35 at distances up to $\sim 10^{2} \mathrm{~nm}$. On the other hand, the zero frequency contribution provides a sizable fraction of the total interaction energy even at distances in the nanometer range if the electric permittivities of the interacting and intervening media are approximately matched in the infrared spectral range. An important example, given its applications in cell biology, is that of lipid layers interacting across an aqueous medium [36, 37, particularly on account of the very large dielectric constant of water at zero frequency. The screening of the van der Waals force was inferred from measurements of the distance between lipid membranes (in the nanometer range) as a function of salt concentration 38. Given the complexity of such systems, comparison with an ab-initio theoretical model for the van der Waals interaction appears as a daunting task.

Simpler configurations, more amenable to theoretical descriptions, could provide a testing ground for inves- 
tigating the salt screening effect. Indeed, the zero frequency contribution dominates the Casimir interaction between polystyrene surfaces across a layer of water even at distances as low as $\sim 10^{2} \mathrm{~nm} 3941$. Unfortunately, in this range the overall attractive signal is weak and a comparison with theory is difficult to implement [42, also in part because of surface roughness effects [43, 44]. Recent force measurements with polystyrene microspheres for distances up to $\sim 20 \mathrm{~nm}$ are not sensitive to screening [45] (see also 46] for a review).

Very weak double-layer forces between polystyrene microspheres, of the order of $10 \mathrm{fN}$, were recently measured with the help of optical tweezers 41. Optical tweezers are ideally suited to probe the zero-frequency contribution to the Casimir interaction, and hence its reduction by salt screening, since trapping with a single laser beam requires a condition of nearly index matching at the laser wavelength (typically in the near infrared). Such experiment should allow for a comparison with theoretical models built on scattering theory.

In this paper, we develop the scattering theory of the Casimir interaction between two parallel planar surfaces separated by a layer of an electrolyte solution. We take the non-local response of the electrolyte into account and analyze the propagation of longitudinal and transverse modes and their coupling by reflection at the surface of the (local) dielectric medium. The Casimir interaction energy is then derived from the matrix describing a round-trip of the electromagnetic waves propagating in between the two interacting surfaces.

When taking typical values for the salt concentration, we find that the presence of ions in solution does not change the contribution of nonzero Matsubara frequencies. For the zero frequency case, we recover the result of Refs. 30 32, which is now reinterpreted as the screened contribution of longitudinal modes written in terms of the corresponding reflection coefficient. We also find an additional term, accounting for the contribution of transverse magnetic (TM) modes at zero frequency.

Our model is based on macroscopic Maxwell equations and constitutive equations for the different materials involved. We take the constitutive equation of a bulk electrolyte to describe its nonlocal response. Note, however, that the surfaces bounding the electrolyte modify the constitutive equations and the derived reflection coefficients 47 50, which should modify our results at distances smaller than the characteristic Debye screening length. Results for the van der Waals interaction beyond the bulk approximation were derived in Ref. [51. A more microscopic theory, built on the analysis of charge fluctuations as in Refs. 52 54, would be required to take into account ion specific effects and density correlations [55, which could play a role at very short distances.

The paper is organized in the following way. In Sec. II we derive the reflection matrix describing the coupling between longitudinal and transverse modes propagating in the electrolyte solution. Such matrix is the building block for developing the scattering formalism of the
Casimir interaction in Sec. III. Numerical results for the case of two polystyrene surface interacting across an aqueous medium are presented in Sec. IV. Sec. V contains concluding remarks.

\section{REFLECTION MATRIX FOR THE ELECTROLYTE-DIELECTRIC INTERFACE}

The key ingredient for the scattering theory to be developed in the next section is the reflection matrix for the interface between the electrolyte solution and the local medium, describing the coupling between longitudinal and transverse magnetic waves. We start by reviewing the hydrodynamical model for 1:1 electrolytes in the bulk approximation [21. In Fourier space, the constitutive equation for the ionic current is

$$
\mathbf{J}(\mathbf{K}, \omega)=\sigma_{\ell}(K, \omega) \mathbf{E}_{\ell}(\mathbf{K}, \omega)+\sigma_{t}(\omega) \mathbf{E}_{t}(\mathbf{K}, \omega),
$$

where $\mathbf{E}_{\ell}(\mathbf{K})$ and $\mathbf{E}_{t}(\mathbf{K})$ are the longitudinal and transverse components of the electric field, respectively. The transverse conductivity is local and given by the usual Drude-like model, whereas the longitudinal conductivity is nonlocal:

$$
\begin{aligned}
\sigma_{t}(\omega) & =\frac{\omega_{P}^{2}}{\gamma-i \omega}, \\
\sigma_{\ell}(K, \omega) & =\frac{\omega_{P}^{2}}{\gamma-i \omega+i v_{\mathrm{th}}^{2} \frac{K^{2}}{\omega}},
\end{aligned}
$$

We have taken $\epsilon_{0}=\mu_{0}=1$. The plasma frequency is $\omega_{P}=\sqrt{N e^{2} / m}$ where $N$ is the number of free charge carriers per volume, $e$ is the electric charge of cations and $m$ is the mass of both cations and anions (assumed to be equal). The nonlocal behavior in real space translates into the $K$ - dependence (spatial dispersion) in (3), which is controlled by the parameter $v_{\mathrm{th}}=\sqrt{k_{B} T / m}$ representing the thermal average velocity of the ions in solution $\left(k_{B}=\right.$ Boltzmann constant).

The electrolyte dielectric functions for transverse and longitudinal waves follow from the conductivities discussed above:

$$
\begin{aligned}
\epsilon_{1}(\omega) & =\epsilon_{b}(\omega)-\frac{\omega_{P}^{2}}{\omega(\omega+i \gamma)} \\
\epsilon_{\ell}(\mathbf{K}, \omega) & =\epsilon_{b}(\omega)-\left(\frac{\omega(\omega+i \gamma)}{\omega_{P}^{2}}-\frac{\lambda_{D}^{2}}{\epsilon_{b 0}} K^{2}\right)^{-1}
\end{aligned}
$$

where $\epsilon_{b}$ is the dielectric function of pure water at zero ionic concentration. We have introduced the Debye screening length in terms of the electrostatic permittivity of the medium $\epsilon_{b 0}$ :

$$
\lambda_{D}=\sqrt{\epsilon_{b 0}} \frac{v_{\mathrm{th}}}{\omega_{P}}=\sqrt{\frac{\epsilon_{b 0} k_{B} T}{N e^{2}}},
$$

which can be tuned by changing the salt concentration $N$. 
The spatial dispersion explicit in Eq. (5) allows for the propagation of longitudinal waves satisfying the dispersion relation

$$
\epsilon_{\ell}\left(\mathbf{K}_{\ell}, \omega\right)=0 .
$$

We write the wave-vector $\mathbf{K}_{\ell}=k_{\ell} \hat{\mathbf{z}}+\mathbf{k}$ in terms of its projection $\mathbf{k}$ on the $x y$ plane. Transverse waves satisfy the standard dispersion relation $\epsilon_{1}(\omega) \omega^{2} / c^{2}=k_{1}^{2}+k^{2}$, with $\mathbf{K}_{t}=k_{1} \hat{\mathbf{z}}+\mathbf{k}$.

We now consider the reflection of longitudinal and transverse waves propagating in the electrolyte by a planar interface perpendicular to the $z$-axis. For a general oblique incidence, TM and longitudinal waves become coupled by reflection, while transverse electric (TE) waves are reflected following the standard Fresnel formula. The frequency $\omega$ and wavevector projection parallel to the surface $\mathbf{k}$ are conserved by reflection.

In Appendix A we derive the reflected fields for a general incident wave propagating from the electrolyte. In addition to the usual boundary conditions for the tangential electric and magnetic fields, we take the condition for the ionic current $J_{z}=0$ at the interface at $z=0$ [21]. We use the indices $s, p$ to represent TE and TM polarizations, respectively. We cast the results in terms of the block-diagonal reflection matrix $\mathcal{R}$ giving the reflected fields as a linear combination of incident tranverse and longitudinal waves:

$$
\begin{gathered}
\left(\begin{array}{l}
E_{s}^{(r)} \\
E_{p}^{(r)} \\
E_{\ell}^{(r)}
\end{array}\right)=\mathcal{R}\left(\begin{array}{c}
E_{s}^{\text {in }} \\
E_{p}^{\text {in }} \\
E_{\ell}^{\text {in }}
\end{array}\right) \\
\mathcal{R}=\left(\begin{array}{ccc}
r_{s s} & 0 & 0 \\
0 & r_{p p} & r_{p \ell} \\
0 & r_{\ell p} & r_{\ell \ell}
\end{array}\right)
\end{gathered}
$$

$r_{s s}$ is the standard Fresnel coefficient for TE polarization, which is not modified by the presence of ions:

$$
r_{s s}=\frac{k_{1}-k_{2}}{k_{1}+k_{2}} \text {. }
$$

The Fresnel coefficient for TM polarization $r_{p p}$ is modified by the coupling with longitudinal waves:

$$
r_{p p}=\frac{\epsilon_{2} k_{1}-\epsilon_{1} k_{2}+\frac{k^{2}}{k_{\ell}} \frac{\epsilon_{2}}{\epsilon_{b}}\left(\epsilon_{1}-\epsilon_{b}\right)}{\epsilon_{2} k_{1}+\epsilon_{1} k_{2}-\frac{k^{2}}{k_{\ell}} \frac{\epsilon_{2}}{\epsilon_{b}}\left(\epsilon_{1}-\epsilon_{b}\right)}
$$

and the diagonal element for longitudinal waves in Eq. (8) is given by

$$
r_{\ell \ell}=\frac{\epsilon_{2} k_{1}+\epsilon_{1} k_{2}+\frac{\epsilon_{2}}{\epsilon_{b}} \frac{k^{2}}{k_{\ell}}\left(\epsilon_{1}-\epsilon_{b}\right)}{\epsilon_{2} k_{1}+\epsilon_{1} k_{2}-\frac{\epsilon_{2}}{\epsilon_{b}} \frac{k^{2}}{k_{\ell}}\left(\epsilon_{1}-\epsilon_{b}\right)}
$$

The nondiagonal matrix elements describe the conversion between TM-polarized and longitudinal waves:

$$
\begin{aligned}
& r_{\ell p}=\frac{2 \frac{k}{k_{\ell}} k_{1} \frac{\epsilon_{2}}{\epsilon_{b}}\left(\epsilon_{1}-\epsilon_{b}\right)}{\epsilon_{2} k_{1}+\epsilon_{1} k_{2}-\frac{k^{2}}{k_{\ell}} \frac{\epsilon_{2}}{\epsilon_{b}}\left(\epsilon_{1}-\epsilon_{b}\right)} \frac{\sqrt{k_{\ell}^{2}+k^{2}}}{\sqrt{\epsilon_{1}} \omega / c} \\
& r_{p \ell}=\frac{2 \epsilon_{2} k}{\epsilon_{2} k_{1}+\epsilon_{1} k_{2}-\frac{k^{2}}{k_{\ell}} \frac{\epsilon_{2}}{\epsilon_{b}}\left(\epsilon_{1}-\epsilon_{b}\right)} \frac{\sqrt{\epsilon_{1}} \omega / c}{\sqrt{k_{\ell}^{2}+k^{2}}}
\end{aligned}
$$

At normal incidence $(k=0)$, the reflection matrix is diagonal, and $r_{p p}$ coincides with the standard Fresnel coefficient $r_{\mathrm{TM}}$ for TM polarization as expected. We also recover the standard TM Fresnel coefficient at frequencies $\omega \gg \omega_{P}$, since $\epsilon_{1} \approx \epsilon_{b}$ according to Eq. (4) in this case. The ions are too slow to couple transverse and longitudinal waves at such large field frequencies, and then the reflection matrix is approximately diagonal. Such property entails that the contribution of nonzero Matsubara frequencies are nearly unaffected by the presence of ions in solution, as discussed in the next section.

\section{ROUND-TRIP MATRIX AND THE CASIMIR INTERACTION ENERGY}

In this section, we derive the Casimir interaction energy between two local dielectric half-spaces separated by a layer (thickness $L$ ) of a (non-local) electrolyte solution, as depicted in Fig. 1. For simplicity, we assume that the local media on both sides have the same electromagnetic properties. Then the reflection matrices at the two interfaces are identical.

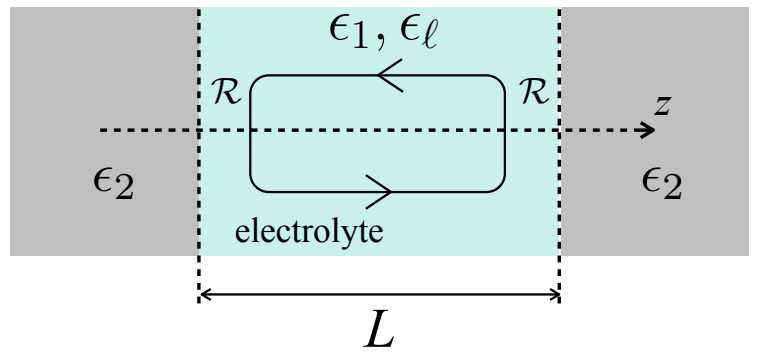

FIG. 1: Casimir interaction across a layer of thickness $L$ containing a non-local electrolyte solution, with dielectric functions $\epsilon_{1}$ and $\epsilon_{\ell}$ for transverse and longitudinal waves, respectively. For simplicity, we assume that the interacting (local) half-spaces share the same dielectric function $\epsilon_{2}$. The Casimir energy is computed from the matrix $\mathcal{R}$ describing the coupling between longitudinal and transverse waves by reflection at the interface.

After a Wick rotation, the Casimir free energy is written as a sum over the Matsubara frequencies $\xi_{n}=$ $2 \pi n k_{B} T / \hbar$, with $n$ integer. The (nonlocal) longitudinal dielectric constant (5) is then written as

$$
\epsilon_{\ell}\left(\mathbf{K},\left|\xi_{n}\right|\right)=\epsilon_{b}\left(i\left|\xi_{n}\right|\right)+\frac{\omega_{P}^{2}}{\left|\xi_{n}\right|\left(\left|\xi_{n}\right|+\gamma\right)+v_{\mathrm{th}}^{2} K^{2}}
$$

Eq. (14) shows that $\epsilon_{\ell}\left(\mathbf{K},\left|\xi_{n}\right|\right)$ is a Lorentzian of width $\approx\left|\xi_{n}\right| / v_{\text {th }}$ for any nonzero Matsubara frequency. In real space, the displacement field $\mathbf{D}$ is then given in terms of the electric field $\mathbf{E}$ by a convolution integral with an exponential kernel, corresponding to the characteristic length scale

$$
\lambda_{n} \equiv v_{\mathrm{th}} /\left|\xi_{n}\right|=\lambda_{\mathrm{dB}} /\left[(2 \pi)^{3 / 2}|n|\right],
$$


where $\lambda_{\mathrm{dB}}=\left(\frac{2 \pi \hbar^{2}}{m k_{B} T}\right)^{\frac{1}{2}}$ is the thermal de Broglie wavelength of the ions at room temperature. Since this length is extremely small, we conclude that the electrolyte behaves approximately as a local medium for all nonzero Matsubara frequencies, as discussed in further detail below. On the other hand, for the zero frequency (14) yields $\epsilon_{\ell}(\mathbf{K}, 0)=\epsilon_{b 0}\left(1+1 /\left(\lambda_{D} K\right)^{2}\right)$, so that the scale of variation with $K$ is now controlled by the Debye screening length $\lambda_{D}$ instead of the de Broglie wavelength. Thus, we expect strong nonlocal effects and the contribution of longitudinal modes as far as the zero-frequency contribution is concerned.

The Casimir free energy is written in terms of the round-trip operator $\mathcal{M}\left(\left|\xi_{n}\right|\right)$ describing the scattering of longitudinal and transverse waves between the interacting surfaces of area $A$ :

$$
\begin{aligned}
\mathcal{F} & =\sum_{n=-\infty}^{\infty} \mathcal{F}_{n} \\
\frac{\mathcal{F}_{n}}{A} & =\frac{k_{B} T}{2} \int \frac{d^{2} k}{(2 \pi)^{2}} \ln \operatorname{det}\left[1-\mathcal{M}\left(\left|\xi_{n}\right|\right)\right]
\end{aligned}
$$

The round-trip matrix $\mathcal{M}\left(\left|\xi_{n}\right|\right)$ is given by

$$
\mathcal{M}\left(\left|\xi_{n}\right|\right)=\mathcal{R} e^{-\mathcal{K} L} \mathcal{R} e^{-\mathcal{K} L}
$$

The reflection matrix $\mathcal{R}$ for the electrolyte-dielectric interface was derived in the previous section. We replace $\omega$ by $i\left|\xi_{n}\right|$ and the axial wave-vector components for the transverse waves in medium $m=1,2$ by $i \kappa_{m}=$ $i \sqrt{k^{2}+\epsilon_{m} \xi_{n}^{2} / c^{2}}$. The longitudinal waves in the electrolyte correspond to

$$
i \kappa_{\ell}=i \sqrt{k^{2}+\frac{1}{v_{\mathrm{th}}^{2}}\left(\left|\xi_{n}\right|\left(\left|\xi_{n}\right|+\gamma\right)+\frac{\omega_{P}^{2}}{\epsilon_{b}\left(i\left|\xi_{n}\right|\right)}\right)} .
$$

The propagation matrix $e^{-\mathcal{K} L}$ is diagonal:

$$
e^{-\mathcal{K} L}=\left(\begin{array}{ccc}
e^{-\kappa_{1} L} & 0 & 0 \\
0 & e^{-\kappa_{1} L} & 0 \\
0 & 0 & e^{-\kappa_{\ell} L}
\end{array}\right)
$$

When writing the scattering formula (17), we have assumed a condition of full thermodynamical equilibrium for all scattering channels, including the longitudinal modes associated to the presence of movable ions. In addition, our derivation is based on the bulk model for the non-local response reviewed in the previous section. Given such assumptions, we are not allowed to take the limit $L \ll \lambda_{D}$, which would eventually suppress the longitudinal channel and introduce the effect of the interfaces already at the level of the constitutive equations.

The determinant in Eq. (17) is evaluated explicitly:

$$
\operatorname{det}\left[1-\mathcal{M}\left(\left|\xi_{n}\right|\right)\right]=\left(1-r_{s s}^{2} e^{-2 \kappa_{1} L}\right) \frac{a_{0}+a_{1} \Delta+a_{2} \Delta^{2}}{(1+\Delta)^{2}}
$$

with $r_{s s}$ defined by Eq. (9). The parameter $\Delta$ quantifies the strength of the coupling between TM and longitudinal waves:

$$
\Delta=\frac{\epsilon_{2}}{\epsilon_{b}} \frac{k^{2}\left(\epsilon_{1}-\epsilon_{b}\right)}{\kappa_{\ell}\left(\epsilon_{2} \kappa_{1}+\epsilon_{1} \kappa_{2}\right)}
$$

and also

$$
\begin{aligned}
a_{0}= & \left(1-e^{-2 \kappa_{\ell} L}\right)\left[1-\left(\frac{\epsilon_{2} \kappa_{1}-\epsilon_{1} \kappa_{2}}{\epsilon_{2} \kappa_{1}+\epsilon_{1} \kappa_{2}}\right)^{2} e^{-2 \kappa_{1} L}\right] \\
a_{1}= & 2\left(1+e^{-2 \kappa_{\ell} L}\right)\left(1+\frac{\epsilon_{2} \kappa_{1}-\epsilon_{1} \kappa_{2}}{\epsilon_{2} \kappa_{1}+\epsilon_{1} \kappa_{2}} e^{-2 \kappa_{1} L}\right) \\
& +\frac{8 \epsilon_{2} \kappa_{1}}{\epsilon_{2} \kappa_{1}+\epsilon_{1} \kappa_{2}} e^{-\left(\kappa_{1}+\kappa_{\ell}\right) L} \\
a_{2}= & \left(1-e^{-2 \kappa_{\ell} L}\right)\left(1-e^{-2 \kappa_{1} L}\right)
\end{aligned}
$$

Since $k_{B} T \gg \hbar \omega_{P}$ for electrolytes, for nonzero Matsubara frequencies we have $\epsilon_{1}-\epsilon_{b} \ll 1$ and then $\Delta \ll 1$. Thus, the round-trip matrix is approximately diagonal leading to independent contributions from the three polarizations:

$$
\begin{gathered}
\frac{\mathcal{F}_{n}}{A} \approx \frac{k_{B} T}{2} \int \frac{d^{2} k}{(2 \pi)^{2}}\left[\sum_{\sigma=s, p} \ln \left(1-r_{\sigma \sigma}^{2} e^{-2 \kappa_{1} L}\right)\right. \\
\left.+\ln \left(1-e^{-2 \kappa_{\ell} L}\right)\right] \quad(n \neq 0)
\end{gathered}
$$

As $\epsilon_{1} \approx \epsilon_{b}$, we can ignore the presence of ions when computing the TM Fresnel reflection coefficients $r_{p p}$ in 23 and use instead the standard result for local dielectric media

$$
r_{p p} \approx \frac{\epsilon_{2} \kappa_{1}-\epsilon_{1} \kappa_{2}}{\epsilon_{2} \kappa_{1}+\epsilon_{1} \kappa_{2}} \quad(n \neq 0)
$$

In addition, we can approximate $\kappa_{\ell} \approx \sqrt{k^{2}+\frac{\xi_{n}^{2}}{v_{\mathrm{th}}^{2}}}$ when computing the contribution of longitudinal modes, corresponding to the second term in the rhs of 23:

$$
\frac{\mathcal{F}_{n}^{\text {long }}}{A} \approx-\frac{k_{B} T}{8 \pi \lambda_{n} L} \exp \left(-\frac{2 L}{\lambda_{n}}\right),(n \neq 0)
$$

where $\lambda_{n}$, defined by Eq. 15], is the characteristic nonlocal length at nonzero Matsubara frequencies. Since $\lambda_{n}$ is smaller than the thermal de Broglie wavelength of the ions and is below the Angström range, $\mathcal{F}_{n}^{\text {long is exponen- }}$ tially suppressed. Thus, we recover the standard DLP result for local materials [17] for all nonzero Matsubara frequencies.

For the zero frequency contribution, we find

$$
\begin{gathered}
a_{0}=a_{2}=\left(1-e^{-2 \sqrt{k^{2}+1 / \lambda_{D}^{2}} L}\right)\left(1-e^{-2 k L}\right) \\
a_{1}=2\left(1+e^{-2 \sqrt{k^{2}+1 / \lambda_{D}^{2}} L}\right)\left(1-e^{-2 k L}\right) .
\end{gathered}
$$


The expression given by 20, then simplifies, and Eq. (17) leads to

$$
\begin{aligned}
& \frac{\mathcal{F}_{0}}{A}=\frac{k_{B} T}{2} {\left[-\frac{\zeta(3)}{8 \pi L^{2}}\right.} \\
&\left.+\int \frac{d^{2} k}{(2 \pi)^{2}} \ln \left(1-r_{\ell \ell}^{2} e^{-2 \sqrt{k^{2}+1 / \lambda_{D}^{2}} L}\right)\right]
\end{aligned}
$$

with the longitudinal reflection coefficient obtained from (11) by taking $\xi=0$ :

$$
r_{\ell \ell}=\frac{\epsilon_{b} \sqrt{k^{2}+1 / \lambda_{D}^{2}}-\epsilon_{2} k}{\epsilon_{b} \sqrt{k^{2}+1 / \lambda_{D}^{2}}+\epsilon_{2} k} \quad(n=0)
$$

The first term in the rhs of 25) accounts for the contribution of TM modes and is written in terms of the value of the Riemann zeta function $\zeta(3) \approx 1.202$. The result $(25)$ can also be obtained directly from the reflection matrix $\mathcal{R}$ by noting that $r_{p \ell} r_{\ell p} \rightarrow 0$ and $r_{p p} \rightarrow-1$ when $\xi \rightarrow 0$.

In conclusion, we find that the modification of the nonzero Matsubara frequency contributions on account of the movable ions is very small. For the zero-frequency case, on the other hand, we find a (screened) contribution from longitudinal waves, the second term in the r.h.-s. of (25), which coincides with the result of Refs. 30 32. Within the scattering approach, such contribution is written in terms of the coefficient $r_{\ell \ell}$ describing the reflection of longitudinal waves at the limit of zero frequency. According to Eq. (25), the screened contribution of longitudinal waves is accompanied by an unscreened contribution from the TM-polarized modes, which is not suppressed even in the limit of strong screening.

\section{A NUMERICAL EXAMPLE: POLYSTYRENE SURFACES INTERACTING ACROSS AN AQUEOUS SOLUTION}

In this section, we apply the formal expressions derived previously to the important example of polystyrene halfspaces interacting across a layer of an aqueous solution. We take $T=293 \mathrm{~K}$ and the Lorentz model with the parameters given by Ref. [56] to describe the required dielectric functions. Similar results are obtained by taking the models proposed in Ref. [40.

It is convenient to define the Hamaker coefficient 40.

$$
H(L)=-12 \pi L^{2} \frac{\mathcal{F}(L)}{A} .
$$

In Fig. 2, we plot $H$ (in units of $k_{B} T$ ) as a function of $L / \lambda_{D}$. We consider two different values for the monovalent salt concentration: $90 \mathrm{mM}$ yielding $\lambda_{D}=1 \mathrm{~nm}$ and $0.9 \mathrm{mM}$ corresponding to $\lambda_{D}=10 \mathrm{~nm}$. They are represented by the black and blue (dark grey) lines in Fig. 2, respectively, which are calculated by combining (16)-(17) with the full exact expression (20). As discussed in connection with Eq. (23), the contribution from nonzero frequencies is well approximated by the DLP standard result
[17. neglecting the presence of ions in solution. For the examples shown in Fig. 2, the relative difference between the exact and DLP results for the nonzero-frequency contribution is of the order of $10^{-5}$.

The red (light grey) line in Fig. 2 corresponds to the separate zero-frequency contribution as computed from Eq. 25). The resulting contribution to the Hamaker coefficient is an universal function of $L / \lambda_{D}$ exhibiting two well defined plateaus, with a crossover at $L / \lambda_{D} \sim 1$. At short distances, $L \ll \lambda_{D}$, we add the contribution of longitudinal channels, whose magnitude is controlled by the reflection coefficient $r_{\ell \ell}$ given by (26), to the universal constant value $H_{0}^{\mathrm{TM}}=\frac{3}{4} \zeta(3) k_{B} T \approx 0.9 k_{B} T$ arising from TM-polarized modes. As the distance increases, the former is suppressed by screening, while the latter defines the asymptotic limit of the total Hamaker coefficient at long distances.

Indeed, as the distance approaches the thermal wavelength $\hbar c /\left(k_{B} T\right)$, the nonzero-frequency contribution is also exponentially suppressed, and then the Hamaker coefficient goes to the zero-frequency asymptotic value $H_{0}^{\mathrm{TM}}$. Such behaviour is indicated in Fig. 2, particularly for the blue (dark grey) curve corresponding to $\lambda_{D}=10 \mathrm{~nm}$, since larger distances are shown in this case. The contribution of nonzero frequencies is maximized at short distances. When added to the zero-frequency value, it defines the unretarded Hamaker 'constant' $H(0)$ corresponding to the short-distance plateau for the black and blue (dark grey) lines. The former, corresponding to $\lambda_{D}=1 \mathrm{~nm}$, develops a second plateau at intermediate distances such that $\lambda_{D} \ll L \ll \lambda_{0}$, with $\lambda_{0}$ representing the typical scale for the resonance wavelengths of water and poystyrene. In this range, the longitudinal zerofrequency term is suppressed by ionic screening, but the nonzero-frequency contribution is still approximately unaffected by electrodynamical retardation. On the other hand, when considering $\lambda_{D}=10 \mathrm{~nm}$, both screening and retardation take place at approximately the same distance range, leading to the more steady decay of the Hamaker coefficient shown by the blue (dark grey) line in Fig. 2.

The results obtained in this section can be directly applied to the interaction between two polystyrene microspheres across an aqueous solution, provided that their radii $R_{1}$ and $R_{2}$ are much larger than the distance. In this case, we can take the proximity force approximation (PFA), also known as Derjaguin approximation [57, in order to derive the attractive Casimir force $F_{\mathrm{SS}}$ between the two spheres from the free energy for parallel planar surfaces taken at the distance of closest approach $L$ :

$$
F_{\mathrm{SS}}=2 \pi R_{\mathrm{eff}} \frac{\mathcal{F}(L)}{A}
$$

with $R_{\mathrm{eff}}=R_{1} R_{2} /\left(R_{1}+R_{2}\right)$.

In Fig. 3, we plot $\left|F_{\mathrm{SS}}\right| / R_{\text {eff }}$ versus distance taking $\lambda_{D}=10 \mathrm{~nm}$. The solid line corresponds to the scattering theory and is obtained from the results for the Hamaker coefficient displayed in Fig. 2 combined with Eqs. (27) 


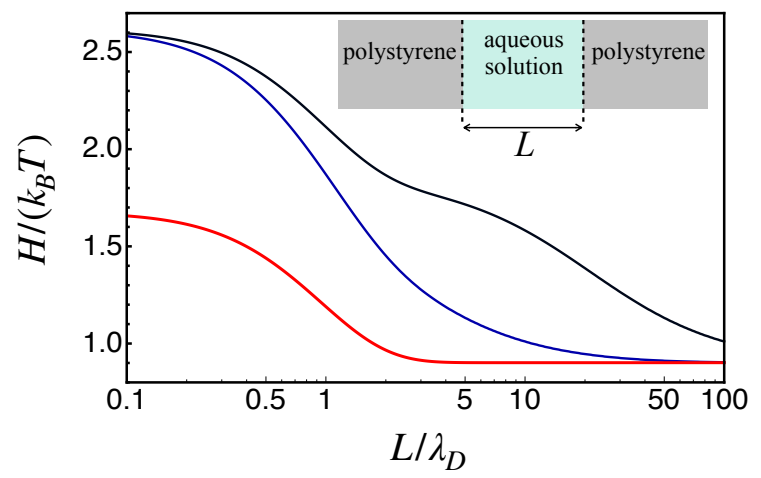

FIG. 2: Variation of the Hamaker coefficient (in units of $k_{B} T$ ) with distance (in units of the Debye screening length $\left.\lambda_{D}\right)$. We consider two polystyrene surfaces interacting across an aqueous solution. Black: $\lambda_{D}=1 \mathrm{~nm}$; blue (dark grey): $\lambda_{D}=10 \mathrm{~nm}$. The red (light grey) line represents the zerofrequency contribution, which is an universal function of $L / \lambda_{D}$.

and (28). The dashed line shows the results calculated from the linear Poisson-Boltzmann equation for the zerofrequency contribution $30-32$, combined with the DLP formalism [17] for the nonzero frequencies. The numerical difference between the two curves arises from the contribution of TM modes at the limit $\xi \rightarrow 0$, which is taken into account within the scattering theory but not by the approach of Refs. 30 32. At short distances, zero-frequency TM contribution is a relatively small fraction of the total interaction energy. Thus, the two curves shown in Fig. 3 are close to each other for $L \lesssim 10 \mathrm{~nm}$, which is the typical range probed with polystyrene colloids [45, 46]. Specifically, the relative discrepancy increases from $35 \%$ at $L=1 \mathrm{~nm}$ to $48 \%$ at $L=10 \mathrm{~nm}$.

On the other hand, Fig. 3 shows that the two models deviate strongly from each other as $L$ increases past the length scales $\lambda_{D}$ and $\lambda_{0}$, due to the suppression of the longitudinal and nonzero-frequency terms as discussed in connection with Fig. 2 An order-of-magnitude discrepancy is found at $L=100 \mathrm{~nm}$. Such range of distances might be reachable by employing optical tweezers [41, which allow for the measurement of very weak interactions.

\section{CONCLUSION}

We have developed the scattering approach to the Casimir interaction across an electrolyte solution. The key ingredient in our formalism is the matrix describing the reflection of transverse and longitudinal waves at the interfaces between the electrolyte and the interacting dielectric materials. Our derivation considers arbitrary frequencies, and the zero-frequency contribution is obtained by taking the limit $\xi \rightarrow 0$ at the very end. As expected, we find that the ions in solution do not modify, to a very

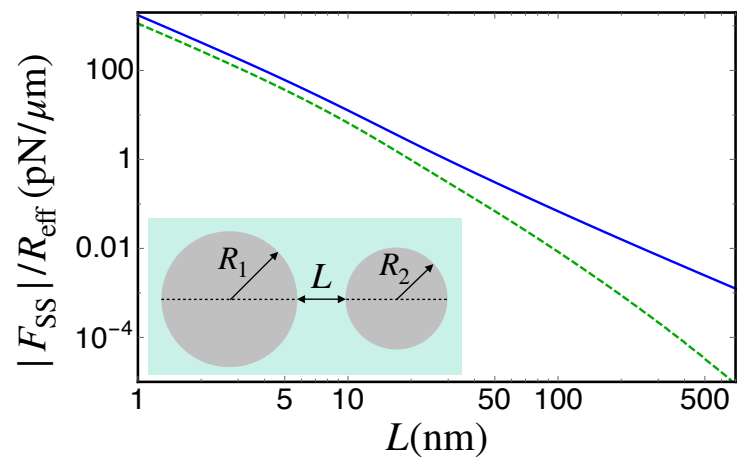

FIG. 3: Variation of the Casimir force $F_{\mathrm{SS}}$ between two polystyrene microspheres with distance $L$. The microspheres of radii $R_{1}$ and $R_{2}$ interact across an aqueous solution with $\lambda_{D}=10 \mathrm{~nm}$. We assume that $R_{1}, R_{2} \gg L$ and calculate the force within the proximity force approximation, which is proportional to the effective radius $R_{\text {eff }}=R_{1} R_{2} /\left(R_{1}+R_{2}\right)$. The dashed line is calculated by considering longitudinal channels at zero frequency alongside the nonzero frequency modes, whereas the solid line also adds TM channels at the limit of zero frequency in accordance with the scattering approach.

good approximation, the contributions at nonzero Matsubara frequencies. At zero frequency, we find a screened contribution of longitudinal scattering channels which agrees with the result of previous derivations based on the linear Poisson-Boltzmann equation [30 32. Within the scattering approach, such screened contribution is cast in terms of the reflection amplitude $r_{\ell \ell}$ for longitudinal waves. Our derivation provides a new insight into the nature of the screened contribution to the Casimir force, and paves the way for the generalization to more general setups and geometries.

In addition to the contribution of longitudinal channels, we find a second contribution at zero frequency, associated to TM-polarized modes, which is not screened by the presence of ions and as a consequence defines the asymptotic behavior of the interaction at long distances.

Our results are based on the bulk model for the electromagnetic response of the electrolyte. Hence they should be valid for distances $L \gtrsim \lambda_{D}$. This condition overlaps with the distance range that allows for the suppression of the double-layer interaction between charged dielectric surfaces. Thus, our derivation is well adapted to experimental conditions aiming at isolating the Casimir interaction from electrostatic force signals.

Acknowledgements. This work has been supported by Centre National de la Recherche Scientifique (CNRS) and Sorbonne Université through their collaboration programs Projet International de Coopération Scientifique (PICS) and Convergence International, respectively. We also acknowledge partial financial support by the Brazilian agencies Conselho Nacional de Desenvolvimento Científico e Tecnológico (CNPq), Coordenação de Aperfeiçoamento de Pessoal de Nível Superior (CAPES), Fundação de Amparo à Pesquisa do Estado 
de Minas Gerais (FAPEMIG), Fundação Carlos Chagas Filho de Amparo à Pesquisa do Estado do Rio de Janeiro (FAPERJ) and Fundação de Amparo à Pesquisa do Estado de São Paulo (FAPESP).

\section{Author contribution statement}

All authors contributed to the development of the theoretical model and the interpretation of the results.

\section{Appendix A: Derivation of the reflection matrix elements}

In this appendix, we derive the matrix $\mathcal{R}$ describing reflection at the interface between the electrolyte and the local medium. We consider longitudinal and transverse waves propagating in the electrolyte occupying the halfspace $z<0$. They are reflected at the interface at $z=0$ and refracted into the local dielectric medium located at the haf-space $z>0$. A similar derivation was presented for describing the optical excitation of plasmons in metals [58. However, we take $J_{z}=0$ at the interface with the electrolyte, whereas Ref. [58] proposes the continuity of $E_{z}$ at the interface between the metallic medium and vacuum as the additional boundary condition. Thus, the reflection matrix derived here diverges from the results of [58] for the metal-vacuum interface.

We first consider the case of an incident TM wave. Without loss of generality, we assume that the incidence plane coincides with the $x z$ plane. The total field in the nonlocal medium 1 is written as $\mathbf{E}=\mathbf{E}_{p}^{(i)}+\mathbf{E}_{p}^{(r)}+\mathbf{E}_{\ell}^{(r)}$ where

$$
\begin{aligned}
\mathbf{E}_{p}^{(i)} & =E_{0} \exp \left(i \mathbf{K}_{p} \cdot \mathbf{r}\right) \hat{y} \times \mathbf{K}_{p} \\
\mathbf{E}_{p}^{(r)} & =r_{p p} E_{0} \exp \left(i \mathbf{K}_{\bar{p}} \cdot \mathbf{r}\right) \hat{y} \times \mathbf{K}_{\bar{p}} \\
\mathbf{E}_{\ell}^{(r)} & =r_{\ell p} E_{0} \exp \left(i \mathbf{K}_{\ell} \cdot \mathbf{r}\right) \mathbf{K}_{\ell}
\end{aligned}
$$

represent the incident, TM reflected, and longitudinal reflected waves, respectively. In the local medium 2 , the refracted field is TM-polarized and given by

$$
\mathbf{E}_{2}=t_{p p} E_{0} \exp \left(i \mathbf{K}_{2} \cdot \mathbf{r}\right) \hat{y} \times \mathbf{K}_{2}
$$

The corresponding wave-vectors are written as

$$
\begin{aligned}
& \mathbf{K}_{p}=\left(k, 0, k_{1}\right) \\
& \mathbf{K}_{\bar{p}}=\left(k, 0,-k_{1}\right) \\
& \mathbf{K}_{\ell}=\left(k, 0,-k_{\ell}\right) \\
& \mathbf{K}_{2}=\left(k, 0, k_{2}\right)
\end{aligned}
$$

We now consider the boundary conditions at the interface $z=0$ between the two media. The continuity of the tangential electric and magnetic fields lead to

$$
\begin{aligned}
\left(1-r_{p p}\right) k_{1}+r_{\ell p} k & =t_{p p} k_{2} \\
\epsilon_{1}\left(1+r_{p p}\right) & =\epsilon_{2} t_{p p}
\end{aligned}
$$

For the third boundary condition, $J_{z}=0$ at $z=0$, we take the dispersion relation (7) into account and then write

$$
\left(\epsilon_{1}-\epsilon_{b}\right)\left(1+r_{p p}\right) k=\epsilon_{b} r_{\ell p} k_{\ell}
$$

We now solve A9, A10 and A11 for the coefficients $r_{p p}, r_{\ell p}$ and $t_{p p}$.

The case of a longitudinal incident wave can be solved in a similar way. Since the dimensionless vectors defining the polarizations in A1 - A3 are non-unitary, the non-diagonal matrix elements describing the coupling between transverse and longitudinal waves should be multiplied by the ratio between the vectors moduli. The final results are given by Eqs. $10-13$.
[1] A. Lambrecht, P. A. Maia Neto, and S. Reynaud, The Casimir effect within scattering theory, New J. Phys. 8, 243 (2006).

[2] S. J. Rahi, T. Emig, N. Graham, R. L. Jaffe, and M. Kardar, Scattering theory approach to electrodynamic Casimir forces, Phys. Rev. D 80, 085021 (2009).

[3] H. B. G. Casimir, On the attraction between two perfectly conducting plates, Proc. K. Ned. Akad. Wet. 51, 793 (1948).

[4] T. Emig, Fluctuation-induced quantum interactions between compact objects and a plane mirror, J. Stat. Mech. (2008) P04007.

[5] P. A. Maia Neto, A. Lambrecht, and S. Reynaud, Casimir energy between a plane and a sphere in electromagnetic vacuum, Phys. Rev. A 78, 012115 (2008).

[6] A. Canaguier-Durand, P. A. Maia Neto, I. Cavero-Pelaez, A. Lambrecht, and S. Reynaud, Casimir Interaction between Plane and Spherical Metallic Surfaces, Phys. Rev. Lett. 102, 230404 (2009).

[7] A. Canaguier-Durand, P. A. Maia Neto, A. Lambrecht, and S. Reynaud, Thermal Casimir Effect in the PlaneSphere Geometry, Phys. Rev. Lett. 104, 040403 (2010).

[8] A. Canaguier-Durand, P. A. Maia Neto, A. Lambrecht, and S. Reynaud, Thermal Casimir effect for Drude metals in the plane-sphere geometry, Phys. Rev. A 82, 012511 (2010).

[9] R. Zandi, T. Emig, and U. Mohideen, Quantum and thermal Casimir interaction between a sphere and a plate: Comparison of Drude and plasma models, Phys. Rev. B 81, 195423 (2010).

[10] M. Hartmann, G.-L. Ingold, and P. A. Maia Neto, Plasma versus Drude Modeling of the Casimir Force: Beyond the Proximity Force Approximation, Phys. Rev. Lett. 119, 043901 (2017).

[11] M. Hartmann, G.-L. Ingold, and P. A. Maia Neto, Advancing numerics for the Casimir effect to experimentally relevant aspect ratios, Phys. Scr. 93, 114003 (2018).

[12] T. Emig, N. Graham, R. L. Jaffe, and M. Kardar, Casimir Forces between Arbitrary Compact Objects, Phys. Rev. Lett. 99, 170403 (2007). 
[13] P. Rodriguez-Lopez, Casimir energy and entropy in the sphere-sphere geometry, Phys. Rev. B 84, 075431 (2011).

[14] S. Umrath, M. Hartmann, G.-L. Ingold, and P. A. Maia Neto, Disentangling geometric and dissipative origins of negative Casimir entropies, Phys. Rev. E 92, 042125 (2015).

[15] M.-T. Jaekel and S. Reynaud, Casimir force between partially transmitting mirrors, J. Phys. I (France) 1, 1395 (1991).

[16] E.M. Lifshitz, The theory of molecular attractive forces between solids Soviet Phys. JETP 2, 73 (1956).

[17] I. E. Dzyaloshinskii, E.M. Lifshitz, and L.P. Pitaevskii, General theory of van der Waals forces, Soviet Phys. Usp. 4, 153 (1961).

[18] C. Genet, F. Intravaia, A. Lambrecht, and S. Reynaud. Electromagnetic vacuum fluctuations, Casimir and Van der Waals forces, Ann. Fondation Louis de Broglie 29, 311 (2004).

[19] J. N. Israelachvili, Intermolecular and Surface Forces (Academic Press, Waltham, 2011).

[20] H. J. Butt and M. Kappl, Surface and Interfacial Forces (Wiley-VCH, Weinheim, 2010).

[21] B. Davies and B. W. Ninham, Van der Waals forces in electrolytes, J. Chem. Phys. 56, 5797 (1972).

[22] E. I. Kats, Influence of nonlocality effects on van der Waals interaction, Sov. Phys. JETP 46, 109 (1977).

[23] R. Esquivel, C. Villareal and W. L. Mochán, Exact surface impedance formulation of the Casimir force: Application to spatially dispersive metals, Phys. Rev. A 68, 052103 (2003). Erratum: Phys. Rev. A 71, 029904E (2005).

[24] R. Esquivel and V. B. Svetovoy, Correction to the Casimir force due to the anomalous skin effect, Phys. Rev. A 69, 062102 (2004).

[25] A. M. Contreras-Reyes and W. L. Móchan, Surface screening in the Casimir force, Phys. Rev. A 72, 034102 (2005).

[26] R. Esquivel-Sirvent, C. Villarreal, W. L. Mochán, A. M. Contreras-Reyes, and V. B. Svetovoy, Spatial dispersion in Casimir forces: a brief review, J. Phys. A: Math. Gen. 39, 6323 (2006).

[27] D. Reiche, M. Oelschläger, K. Busch, and F. Intravaia, Extended hydrodynamic description for nonequilibrium atom-surface interactions, J. Opt. Soc. Am. B 36, C53 (2019).

[28] G. Barton, Some surface effects in the hydrodynamic model of metals, Rep. Prog. Phys. 42, 65 (1979).

[29] L. M. Woods, D. A. R. Dalvit, A. Tkatchenko, P. Rodriguez-Lopez, A. W. Rodriguez, and R. Podgornik, Materials perspective on Casimir and van der Waals interactions, Rev. Mod. Phys. 88, 045003 (2016).

[30] D. J. Mitchell and P. Richmond, A General Formalism for the Calculation of Free Energies of Inhomogeneous Dielectric and Electrolyte System, J. Colloid Interface Sci. 46, 118 (1974).

[31] J. Mahanty and B. W. Ninham, Dispersion Forces (Academic, London) 1976.

[32] V. A. Parsegian, Van der Waals Forces: A Handbook for Biologists, Chemists, Engineers, and Physicists (Cambridge, New York) 2006.

[33] N. G. Van Kampen, B. R. A. Nijboer and K. Schram, On the Macroscopic Theory of Van der Waals Forces, Phys. Lett. 26A, 307 (1968).

[34] J. Munday, F. Capasso, V. A. Parsegian and S. M.
Bezrukov, Measurements of the Casimir-Lifshitz force in fluids: The effect of electrostatic forces and Debye screening, Phys. Rev. A 78, 032109 (2008).

[35] A. Le Cunnuder, A. Petrosyan, G. Palasantzas, V. Svetovoy, and S. Ciliberto, Measurement of the Casimir force in a gas and in a liquid, Phys. Rev. B 98, 201408R (2018).

[36] V. A. Parsegian and B. W. Ninham, Temperaturedependent van der Waals forces, Biophy. J. 10, 664 (1970).

[37] V. A. Parsegian and B. W. Ninham, Toward the Correct Calculation of van der Waals Interactions Between Lyophobic Colloids in an Aqueous Medium, J. Colloid Interface Sci. 37, 332 (1971).

[38] H. I. Petrache, Th. Zemb, L. Belloni, and V. A. Parsegian, Salt screening and specific ion adsorption determine neutral-lipid membrane interactions, PNAS 103, 7982 (2006).

[39] V. A. Parsegian, Long range van der Waals forces, in Physical Chemistry: enriching topics in colloid and surface science, edited by $\mathrm{H}$. van Olphen and K. J. Mysels (Theorex, 1975).

[40] W. B. Russel, D. A. Saville and W. R. Schowalter, Colloidal Dispersions (Cambridge University Press, Cambridge, 1989).

[41] D. S. Ether jr. et al., Probing the Casimir force with optical tweezers, EPL 112, 44001 (2015).

[42] M. A. Bevan and D. C. Prieve, Direct Measurement of Retarded van der Waals Attraction, Langmuir 15, 7925 (1999).

[43] P. A. Maia Neto, A. Lambrecht, and S. Reynaud, Casimir effect with rough metallic mirrors, Phys. Rev. A 72, 012115 (2005).

[44] P. J. van Zwol, G. Palasantzas, and J. Th. M. De Hosson, Influence of random roughness on the Casimir force at small separations Phys. Rev. B 77, 075412 (2008).

[45] M. Elzbieciak-Wodka, M. N. Popescu, F. J. M. RuizCabello, G. Trefalt, P. Maroni, and M. Borkovec, Measurements of dispersion forces between colloidal latex particles with the atomic force microscope and comparison with Lifshitz theory, J. Chem. Phys. 140, 104906 (2014).

[46] G. Trefalt, Th. Palberg, and M. Borkovec, Forces between colloidal particles in aqueous solutions containing monovalent and multivalent ions, Curr. Opinion Colloid Interf. Sci. 27, 9 (2017).

[47] G. S. Agarwal, D. N. Pattanayak, and E. Wolf, Structure of the electromagnetic field in spatially dispersive medium, Phys. Rev. Lett. 27, 1022 (1971).

[48] G. S. Agarwal, D. N. Pattanayak, and E. Wolf, Refraction and reflection on a spatially dispersive medium, Opt. Commun. 4, 255 (1971).

[49] G. S. Agarwal, D. N. Pattanayak, and E. Wolf, Boundary conditions on exciton polarization and mode coupling in a spatially dispersive medium, Opt. Commun. 4, 260 (1971).

[50] A. A. Maradudin and D. L. Mills, Effect of spatial dispersion on the properties of a semi-infinite dielectric, Phys. Rev. B 7, 2787 (1973).

[51] V. N. Gorelkin and V. P. Smilga, Calculation of Intermolecular Interaction Forces between Bodies Separated by a Film of a Strong Electrolyte Solution, Soviet Phys. JETP 36, 761 (1974).

[52] J. Sarabadani, A. Naji, D. S. Dean, R. R. Horganm and R. Podgornik, Nonmonotoic fluctuation-induced interac- 
tions between dielectric slabs carrying charge disorder, J. Chem. Phys. 133, 174702 (2010).

[53] D. S. Dean, V. A. Parsegian, and R. Podgornik, Fluctuation of thermal van der Waals forces due to dipole fluctuations, Phys. Rev. A 87, 032111 (2013).

[54] D. S. Dean and R. Podgornik, Relaxation of the thermal Casimir force between net neutral plates containing Brownian charges, Phys. Rev. E 89, 032117 (2014).

[55] Eletrostatics of soft and disordered matter, edited by D. S. Dean, J. Dobnikar, A. Naji and R. Podgornik (Pan
Stanford, Singapore, 2014).

[56] P. J van Zwol and G. Palasantzas, Repulsive Casimir forces between solid materials with high-refractive-index intervening liquids, Phys. Rev. A 81, 062502 (2010).

[57] B. Derjaguin, Untersuchungen über die Reibung und Adhäsion, IV - Theorie des Anhaftens kleiner Teilchen, Kolloid-Zs. 69, 155 (1934).

[58] A. R. Melnyk and M. J. Harrison, Theory of optical excitation of plasmons in metals, Phys. Rev. B 2, 835 (1970). 